Revue scientifique sur la conception et l'aménagement de l'espace

$8 \mid 2012$

Les concepteurs de jardins et de parcs japonais

\title{
Participation et développement soutenable des territoires
}

L'expérience de médiateur de Kuwako Toshio au Japon

Participation and Sustainable Development of Territories - The Experience of Mediator of Kuwako Toshio in Japan

\section{Alban Mannisi}

\section{OpenEdition}

Journals

Édition électronique

URL : http://journals.openedition.org/paysage/16019

DOI : $10.4000 /$ paysage. 16019

ISSN : 1969-6124

\section{Éditeur :}

École nationale supérieure du paysage de Versailles-Marseille, Institut national des sciences appliquées Centre Val de Loire - École de la nature et du paysage, École nationale supérieure d'architecture et de paysage de Bordeaux, École nationale supérieure d'architecture et de paysage de Lille, Agrocampus Angers

Référence électronique

Alban Mannisi, «Participation et développement soutenable des territoires », Projets de paysage [En ligne], 8 | 2012, mis en ligne le 13 juillet 2012, consulté le 11 mars 2021. URL : http://

journals.openedition.org/paysage/16019; DOI : https://doi.org/10.4000/paysage.16019

Ce document a été généré automatiquement le 11 mars 2021.

Projets de paysage 


\title{
Participation et développement soutenable des territoires
}

\author{
L'expérience de médiateur de Kuwako Toshio au Japon \\ Participation and Sustainable Development of Territories - The Experience of \\ Mediator of Kuwako Toshio in Japan
}

Alban Mannisi

1 La participation de la société civile devient un enjeu majeur du développement soutenable des territoires au $\mathrm{xxI}^{\mathrm{e}}$ siècle. Au niveau international, cette volonté d'implication des communautés locales se trouve énoncée dans la déclaration de Rio (1992), qui souligne que «la meilleure façon de traiter les questions d'environnement est d'assurer la participation de tous les citoyens concernés, au niveau qui convient ${ }^{1}{ }$. De même, la Convention européenne du paysage (art. 5), signée à Florence en 2000, souligne la nécessité de mettre en place des procédures de participation du public, des autorités locales et régionales ainsi que des autres acteurs concernés par la conception et la réalisation des politiques du paysage afin de favoriser une prise en compte des différentes perceptions des parties de territoire par les populations.

2 En France, une série de lois insiste sur l'importance d'associer les populations locales à travers des processus de consultation, de concertation et de débat public. Certaines s'inscrivent dans la logique des déclarations internationales citées précédemment, d'autres cherchent à répondre à des demandes hexagonales². En 2007, le Grenelle de l'environnement a rappelé l'intérêt de la participation du public et l'importance de «nouvelles formes de gouvernance favorisant la mobilisation de la société par la médiation et la concertation » pour construire/s'orienter vers une/la controversée démocratie écologique ${ }^{3}$.

3 À l'étranger et au sein d'une société aussi différente de la France qu'est le Japon, on trouve également une montée en puissance des phénomènes participatifs. Dans le cas de l'archipel nippon, ces derniers viennent souvent en réaction à de nombreux aménagements territoriaux effectués à bas coût après la Seconde Guerre mondiale et ne prenant pas en compte ni l'environnement existant ni les populations locales. Des lois 
récentes mettent en évidence ces évolutions. Ainsi, en 1998, la loi $\mathrm{NPO}^{4}$ ( $H i$ shūeki soshiki-hô) favorise la création d'associations civiles investies dans les affaires de la communauté. Dans cet élan de légitimation politique des actions citoyennes, la problématique participative rencontre la gestion environnementale.

En 1998, faisant face aux incessantes querelles autour d'inondations et de projets de rétablissement hydraulique, le gouvernement japonais met en place la loi sur les cours d'eau (kasen-hô), qui déclenche les procédures participatives dans la délicate mise en œuvre de restauration du réseau hydraulique, entièrement bétonné sur l'archipel. Cette participation s'inscrit dans le cadre d'une décentralisation vers les communes de faible envergure grâce à la loi d'autonomie des communautés de petites tailles (Chiho bunken ikkatsu hô) en 2000 et la restructuration du pouvoir vers des ministères modernisés, soucieux d'intégrer les points de vue des particuliers dans de larges projets de construction publique. En 2004 sera ratifiée la loi paysage (Keikan hố).

5 C'est au cœur de cette dynamique que le gouvernement japonais a fait appel, au cours des années 1990, à des groupes multidisciplinaires de recherche. Ceux-ci favorisent la prise de parole de la population afin d'anticiper et de résoudre les controverses environnementales dans le cadre de projets territoriaux (infrastructures, création de barrage, contrôle des rives des rivières, etc.). Le philosophe japonais Kuwako Toshio, professeur à l'université technologique de Tōkyō, sert souvent de médiateur.

Chercheur associé au sein du laboratoire de recherche d'éthique environnementale, nous y étudions les nouvelles pratiques d'aménagements paysagers en prenant en compte l'essor des éthiques écologiques. Dans cette étude, nous nous intéresserons à la posture nouvelle que des médiateurs, tels que le philosophe environnementaliste Kuwako Toshio, produisent en terme de construction du paysage. Cet article aborde un aspect de sa contribution philosophique et un cas pratique d'implication dans l'établissement de "construction d'un consensus» entre les différents points de vue des habitants, des maîtres d'œuvre et des politiques' ${ }^{6}$.

\section{Kuwako Toshio et le portrait du lieu (Kûkan no rireki)}

7 L'engagement de Kuwako Toshio s'inscrit dans un parcours personnel qu'il est nécessaire de retracer pour expliquer les raisons qui l'ont conduit à s'intéresser aux questions environnementales et au phénomène participatif.

\section{Kuwako Toshio, l'engagement via la voie philosophique}

8 Né en 1951, il grandit au cœur de la préfecture de Gunma, une région centrale de l'archipel présentant une variété considérable de sites anciens et d'agglomérations réputées pour leurs sources thermales. Au sortir de la guerre, les projets de construction constituant un vecteur crucial de relance de l'économie, le gouvernement lance de grands projets qui bouleversent considérablement l'environnement naturel et humain du pays. En 1964 notamment, la précipitation avec laquelle les Jeux olympiques sont organisés à Tōkyō et l'absence d'expérience en matière de construction de cette ampleur chez les bâtisseurs japonais provoquent une réelle désorganisation. La rivière Ara, qui traverse Tōkyō depuis la préfecture de Gunma, devient le théâtre d'extractions sauvages de sable. Des fossés creusés sans aucune mesure de protection causent de nombreux accidents. Le changement brutal de l'usage du paysage fluvial, jusqu'alors en 
mutation lente, bouleverse le jeune Kuwako Toshio, habitué à jouer le long de la rivière Ara. Il s'interroge sur les causes qui peuvent entraîner une société à détruire son propre environnement. Pour répondre à cette question, il décide, après son baccalauréat, de se diriger vers des études de philosophie.

Dans les années 1970, époque à laquelle il débute sa formation, l'enseignement de ce domaine au Japon et les questionnements sur l'être et l'existence se fondent principalement sur des classiques occidentaux. Toutefois, en 1983, lors d'un voyage d'étude en Grèce, il mesure le fossé entre les cultures européenne et japonaise. Pour trouver des réponses aux bouleversements que connait l'archipel nippon, il change la direction de sa recherche et réinterroge les fondements de sa propre culture. Aussi décide-t-il de relire les penseurs chinois et japonais. Cette quête l'amène à publier, en 1996, un livre intitulé Kisō no Tetsugaku (Philosophie du changement et de la transformation) (Kuwako, 1996), qui propose une exploration de l'être ancré dans l'espace de vie japonais. Cette première publication sera suivie de l'essai Kankyô no Tetsugaku (Une philosophie de l'environnement) en 1999 (Kuwako, 1999a), où il étudie quelques lieux emblématiques japonais influencés par la philosophie chinoise. En visitant quelquesuns des sites les plus significatifs de l'influence des penseurs néoconfucéens sur le milieu japonais, son chemin croise celui de Saigyô Hôshi (1118-1190), poète et moine anachorète. C'est une découverte capitale (Kuwako, 1999b). Marchant sur les pas du moine voyageur et relisant ses poèmes in situ, Kuwako Toshio revit les espaces transcrits par la poésie. De ses investigations, il conclut à la nécessité de prendre en compte l'environnement naturel en lien avec les interactions des êtres humains lors de travaux d'aménagement territoriaux.

Suite à cette publication, en 2001, l'universitaire est convié par le gouvernement à participer à des groupes de recherche et à formuler des propositions de méthodologie auprès des ministères alors en pleine refonte, dont le ministère du Territoire, de l'Infrastructure, des Transports et du Tourisme. Dans ce but, et sous la tutelle des programmes gouvernementaux japonais, il suit des formations américaines afin d'étudier l'élaboration de consensus (consensus Building) (Susskind, 201077).

11 À la suite de cette première initiation, Kuwako Toshio est directement impliqué dans la médiation de conflits entre experts, gouvernement et société civile, au cours des programmes de recherche engagés dans le cadre de la nouvelle loi sur le réaménagement des rivières (1998). Mais il lui apparaît très vite que les modalités d'implication des sociétés civiles visent essentiellement à éluder toute controverse. Selon lui, ces nouvelles dispositions participatives engendrant une multiplicité des points de vue (et parfois la division au sein même d'une communauté initialement unie par le conflit) sont elles-mêmes sources de nouvelles entraves au projet de résolution qu'elles étaient pourtant censées réduire (Kuwako, 2008). Le philosophe intègre ainsi la nécessité de considérer l'élaboration du paysage comme une "construction sociale $^{8}$ " (Berger, Luckmann, 2006) ; comme pour toute construction, il en note la fragilité et le constant travail de cohésion que cela suppose (Kuwako, 2009). De plus, il entreprend de développer une recherche quant à la structuration d'un tel consensus approprié au Japon. Mais plus encore - et c'est ce qui rend son approche si pertinente -, il élabore un procédé capable de rendre compte des raisons sous-jacentes générant des litiges, ce qu'il appelle le profil historique (ou la généalogie) de ces raisons au cours duquel s'établit la fabrication du lieu. C'est ce qu'il va théoriser avec le kûkan no rireki. 


\section{Le concept de kûkan no rireki}

12 Kûkan no rireki, que l'on peut traduire par curriculum (rireki) spatial (kûkan), est une méthode permettant d'apprécier la temporalité - l'historialité (Heidegger, 1986) observable à partir des traces visibles d'un espace. C'est ce que Kuwako Toshio désigne également par "portrait du lieu", que le terme curriculum traduit plus exactement pour qualifier la relation temporelle que le sujet citoyen entretient avec le passé. Tout comme les points de vue des habitants qui vont émerger au sein de projets participatifs, le lieu résume les actions des êtres qui l'ont habité (Augé, 1992). Il contient, à la manière des palimpsestes, ses raisons dissimulées en profondeur. L'idée repose sur une acception précise de la notion d'espace, kûkan. Celui-ci est déterminé par sa relation au temps et à l'action du corps qui, s'il est localisé, demeure, comme le précise le philosophe, une abstraction considérée dans ses dynamiques agissantes entre géographie et perception/action de l'être. Afin d'établir une approche constructive de la perception de l'espace et ainsi mieux saisir son déploiement, le philosophe interroge donc essentiellement les dynamiques permettant de rendre compte de cette construction.

Dans un premier temps, ce principe permet de saisir la fabrication du paysage dans le sens d'une production du territoire par une société en son exercice politique (le politique ${ }^{9}$, pris au sens de l'organisation d'une société sur son socle géographique). Dans un deuxième temps, et parce que cette méthode s'est nourrie des projets participatifs auxquels le philosophe a collaboré, il s'agit de permettre, aux divers sujets citoyens qui interviennent dans un conflit, d'assumer leur part de responsabilité dans l'établissement de ces lieux de vie. Le kûkan no rireki permet de saisir, par le biais du temps et de l'action, le caractère constructif (façonné par l'homme) que révèlent nos espaces. Inspiré par le moine philosophe Saigyô (le poète) et par sa formation au consensus building américain (avec ses spécificités japonaises), Kuwako Toshio procède par allers-retours entre recherches théoriques et applications pratiques pour perfectionner en permanence son outil de médiation environnementale.

14 Ainsi, œuvrant au sein de différents projets de grande ampleur comme le rééquilibrage du débit de la rivière Ohashi, il entame en 2005 un projet dialogique ${ }^{10}$ particulièrement réussi: la requalification de la rue qui mène au temple de la ville d'Izumo, IzumoTaisha. Entamées en juillet 2010, les discussions entre les experts et la population, qui se poursuivent aujourd'hui, nous aideront à clarifier cette éthique en action.

\section{La requalification de la voie menant au temple d'Izumo-Taisha : un exemple de démarche participative}

15 Izumo-Taisha est l'un des plus anciens sanctuaires japonais. Il fait partie de la préfecture de Shimane, sur la côte est du Japon, un domaine patrimonial particulièrement spectaculaire. La région comprend nombre d'héritages prestigieux temples, parcs et canaux - mais elle est également pourvue d'un réseau hydraulique complexe qui offre à sa capitale Matsue et à la ville d'Izumo la beauté de ses rivières, lacs et sources. En même temps que des inondations remarquables. 
16 Face à cette problématique ambivalente, le gouvernement japonais a répondu favorablement aux demandes d'élus locaux d'organiser des réunions de médiation pour sortir de conflits récurrents ${ }^{11}$ entre associations citoyennes et ingénierie civile. Préserver cette zone spectaculaire ${ }^{12}$ de futures inondations nécessite un vaste plan de travaux, prévus sur vingt ans (2005-2025). La médiation de Kuwako Toshio qui, en 2005, a rejoint le comité du développement des alentours de la rivière Ohashi, prend alors toute son efficacité: il organise des forums de consensus et intervient plus particulièrement sur le délicat projet de requalification de l'axe qui mène au prestigieux sanctuaire d'Izumo-Taisha.

17 Trésor national depuis 1952, le sanctuaire attire toujours un nombre considérable de visiteurs, ce qui conduit les autorités locales à réorganiser le circuit routier attenant au domaine. Depuis l'ancien village-rue, une promenade bordée d'arbres majestueux monte en direction du sanctuaire adossé à un massif montagneux densément boisé. Afin de désengorger les infrastructures et l'urbanisation inadaptées au flot de cars touristiques, un parking proche du site a été aménagé, permettant aux touristes un accès direct. Suite à cet aménagement, comme le parc ne se prolonge plus vraiment jusqu'à la rue commerçante de la ville, en raison d'une promiscuité voitures/piétons dangereuse, les commerces ont périclité (figure 1).

Pour rétablir une liaison piétonne allant du village jusqu'au sanctuaire, les ingénieurs civils proposent un élargissement de la voirie aux abords des commerces afin d'harmoniser les circulations d'automobiles et de piétons. En raison de l'irrégularité des façades, le plan initial, très «impactant » (figure 4), prévoyait la destruction de nombreux commerces dont il était justement censé ralentir la disparition. La protestation véhémente des citoyens ${ }^{13}$ et leur refus de négocier nécessitèrent l'intervention d'un médiateur entre des experts en ingénierie et une population qui ne se comprennent pas plus qu'ils ne s'écoutent. Kuwako Toshio est alors mandaté afin de renouer le contact entre les acteurs de la revalorisation de ce paysage spectaculaire (figure 2). 
Figure 1. Le projet de requalification du site d' Izumo-Taisha
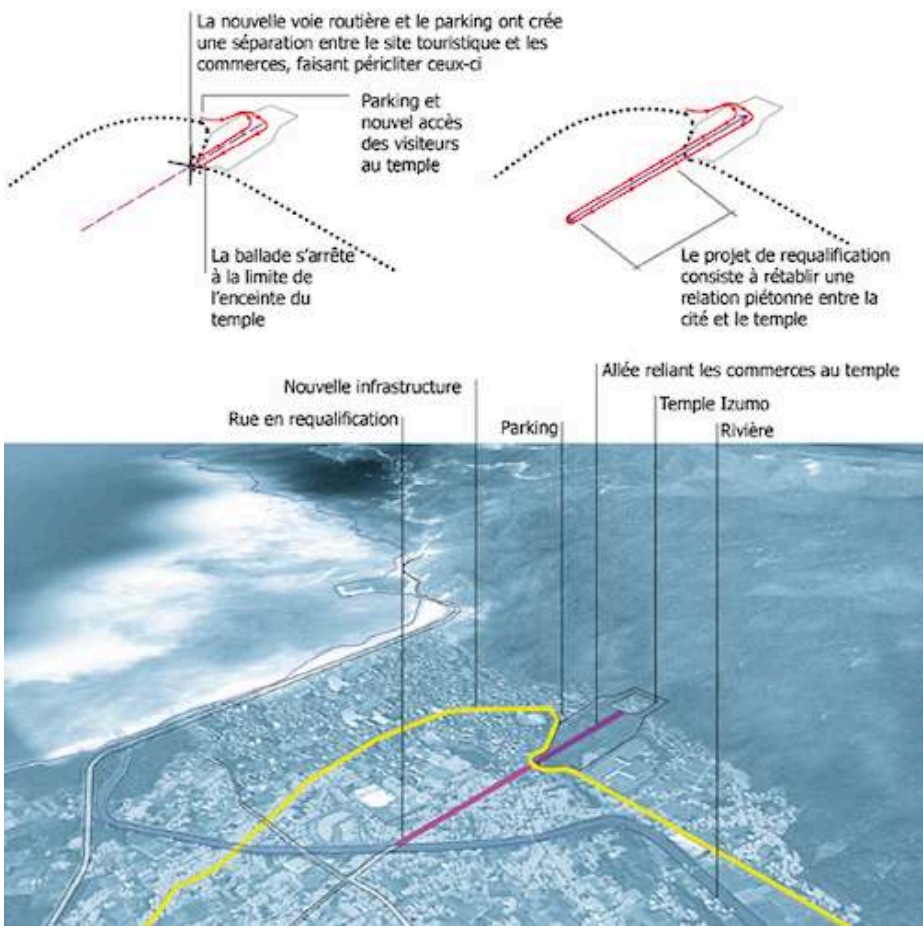

Le village-rue qui découle de l'allée du temple n'est désormais plus emprunté à cause de la nouvelle voie qui disjoint les deux espaces piétons.

Source : Alban Mannisi.

Figure 2. Vue en direction de la rue commerçante depuis l'allée du temple Izumo-Taisha

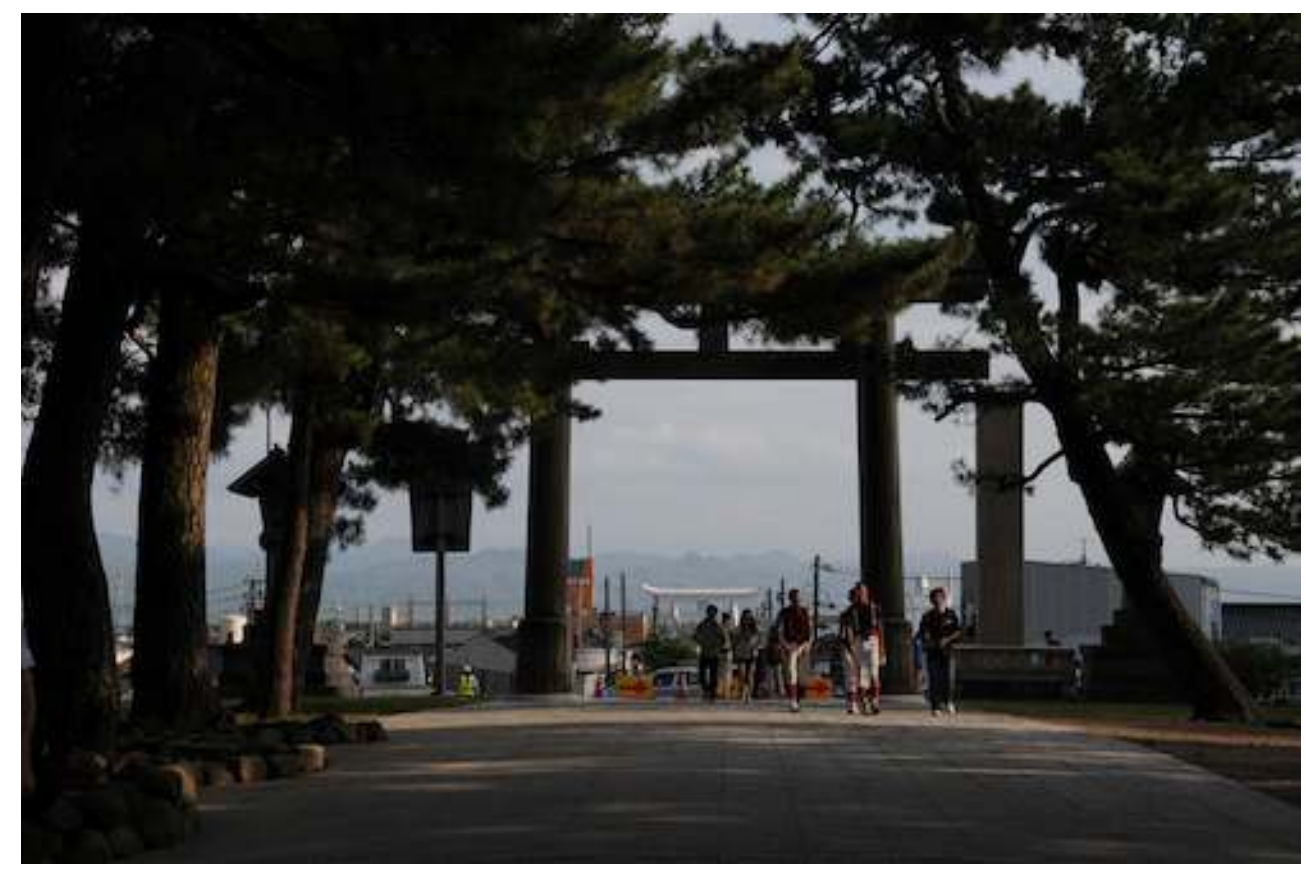

Source : Kuwako Toshio 

dynamique de l'espace, encore faut-il pouvoir le mettre en œuvre avec les acteurs locaux. Les discussions qui permettent de diagnostiquer l'usage conflictuel des lieux doivent donc être clairement organisées afin de révéler de façon juste l'action des citoyens au sein des espaces et de rédiger ce que l'on peut appeler une note d'intention. Une grande importance est donnée à la communication du consensus opéré. À chaque fin de réunion, tout acteur doit repartir avec une idée claire des informations émises. C'est le travail de synthèse fait par le médiateur (figure 3). Mettre en place cette philosophie environnementale afin de canaliser les énergies éparses et comprendre avec les intéressés ce que recèle cet espace vécu (Fremont, 1976) permet l'émancipation des acteurs locaux fragilisés par l'autorité brutale de projets comme celui d'Izumo.

Figure 3. Réunion entre les habitants de la rue, les experts (éclairagiste, urbaniste, paysagiste), et le médiateur Kuwako Toshio s'exprimant à l'assemblé à Izumo le 27 août 2011

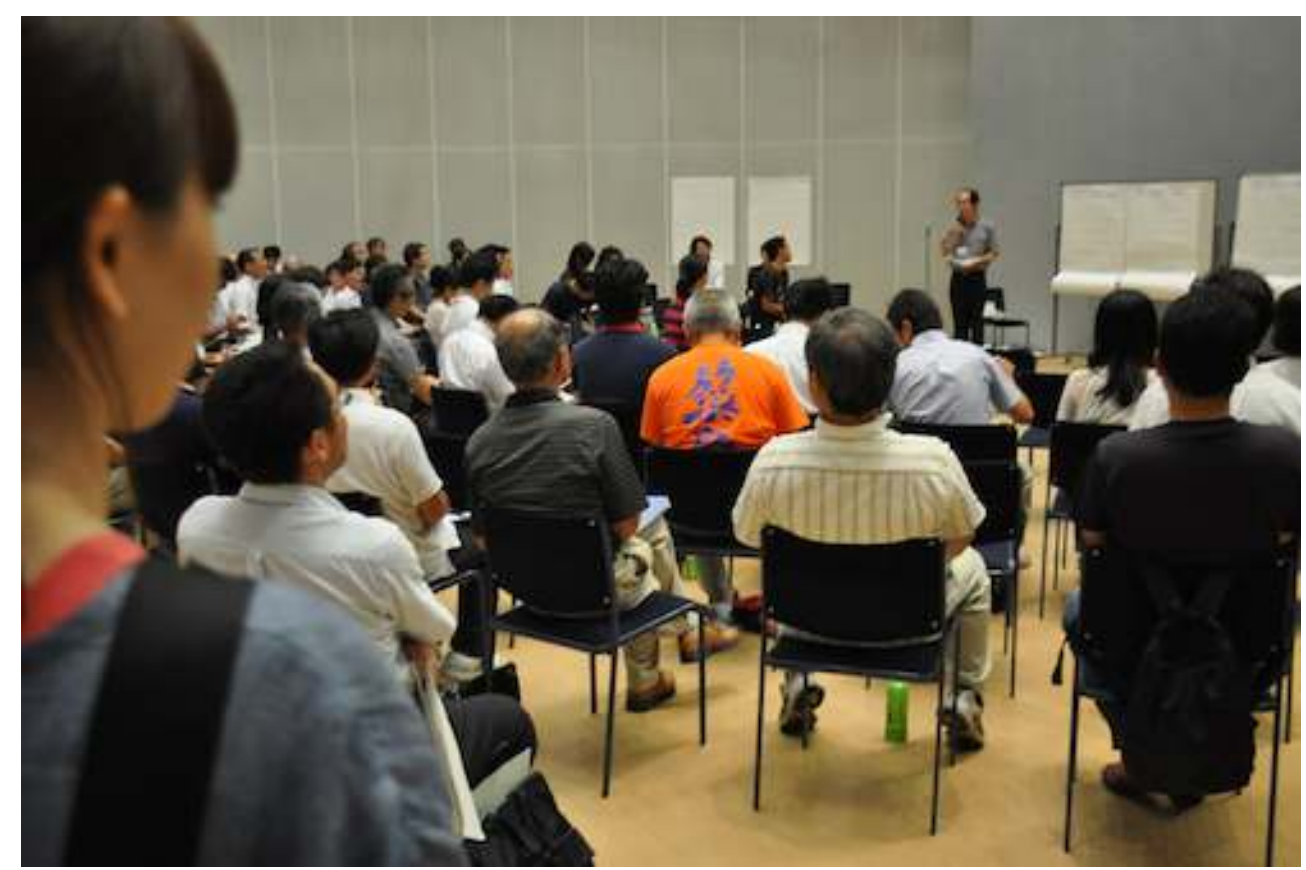

Source : Alban Mannisi.

En premier lieu, le rôle de Kuwako Toshio est celui imparti à tout leader de projet de participation citoyenne dans l'aménagement du territoire: permettre aux experts, urbanistes, paysagistes et ingénieurs civils d'entendre les aspirations encore informulées des habitants de la commune. Mais, comme il le précise, il ne s'agit pas d'une conciliation, qui se résumerait à faire coïncider des propositions divergentes alors que celles-ci ne sont généralement pas encore consciemment formulées. La médiation ne prend son sens et n'a de pérennité que si les habitants s'approprient et assument les dynamiques de leur futur cadre de vie. 


\section{Les étapes de la médiation}

21 Kuwako Toshio, très attentif à la communication spatiale (kûkan no komyunikêshon), procède en plusieurs étapes (Kuwako, 2007).

1. Dans un premier temps, le comité décisionnel ${ }^{14}$ doit émettre une proposition, afin que les enjeux du débat soient clairement formulés, à savoir les passifs et actifs d'un projet qui peuvent créer la controverse, bien souvent née de zones d'ombre. Face à la complexité des projets territoriaux et à la multitude des agents qui prennent part à la controverse (véhiculant chacun une vision du problème), informer l'ensemble des groupes investis des enjeux est une étape longue et cruciale. Elle doit déboucher sur un moment clé d'assentiment, une étape clairement comprise, appelée natoku.

2. Vient ensuite le moment du forum de consensus, qui tient compte de la particularité du peuple japonais dont la coutume est de ne pas donner son opinion frontalement mais plutôt de façon parcimonieuse pour ne pas compromettre l'unité du groupe (villages, entreprises, nation). Cette particularité de se concevoir comme un tout, qui produit un évitement du conflit par omission, est appelée oboro dans la région d'Izumo. Pour ne pas compromettre cet équilibre, on procède de façon semi-anonyme : chacun note son opinion puis les Post-it sont affichés et commentés en groupe. Cet espace de communication où l'attention est portée sur un tiers est appelé communication indirecte (kansetsu-teki komyunikēshon) (figure 3). Kuwako Toshio rapproche ces espaces de collaboration des cérémonies du thé (chanoyu) ou des arrangements floraux (ikebana), où l'espace constitue lui-même cette spirale d'échanges entre les êtres.

3. Vient alors la rédaction des procédures du forum, afin que la révélation de ce curriculum spatial qui expose la «construction sociale» du lieu (Berger, 2006) soit lisible ultérieurement. C'est l'acceptation de la controverse, comme phase productive dans l'établissement d'un projet communautaire (Tricot, 1994).

L'ensemble de ces étapes a pour résultat de rétablir la confiance mutuelle entre les différents acteurs de la communauté et de permettre ainsi, via le lien social (kizuna), de renforcer l'environnement social (figure 5). 
Figure 4. Discussion du choix des matériaux in situ

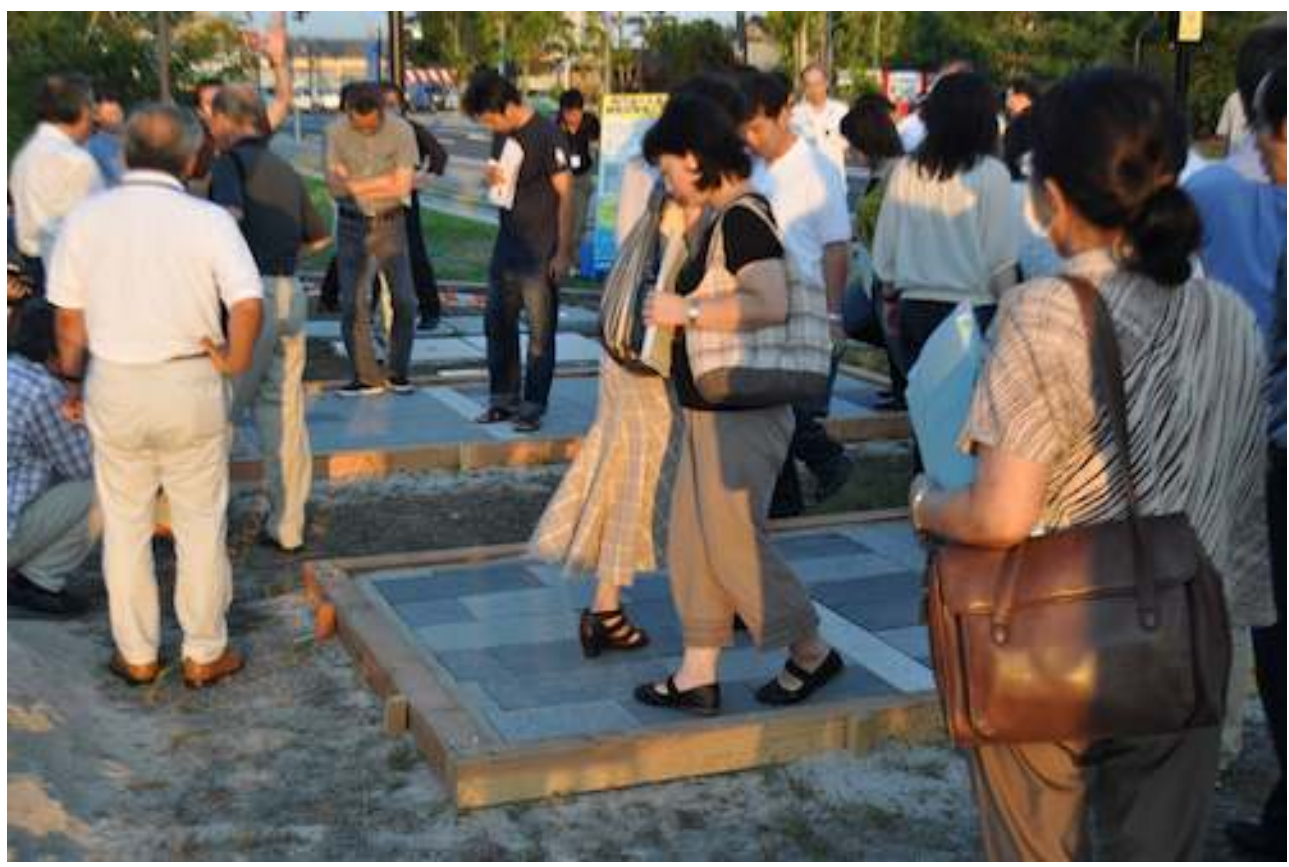

Source : Alban Mannisi.

Figure 5. Discussion in situ de la pertinence des propositions d'aménagement

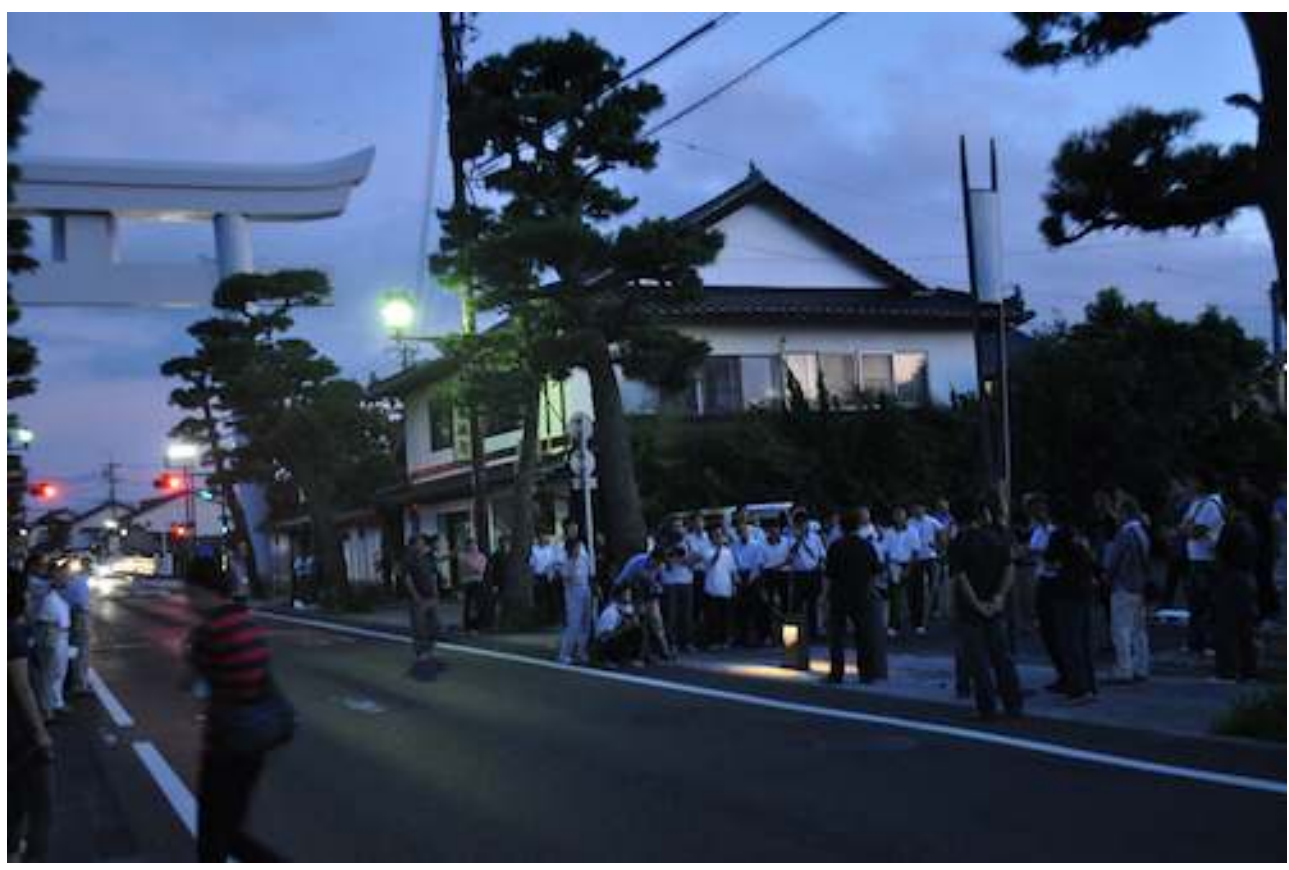

Source : Alban Mannisi.

23 À Izumo, cette participation active a conduit l'ensemble des parties investies à invalider la proposition initiale des experts urbanistes qui, en élargissant la voie, « impactait » le bâti (village-rue) et vouait quantité de commerces à la démolition (figure 6). Le plan adopté «n'impactera» donc pas les commerces que le projet de requalification de la 
voie est censé préserver. Dès lors, en découleront les différentes modalités de séparation au sol des espaces partagés entre véhicules et piétons (figure 7).

Chaque nouvelle étape du processus d'acceptation du consensus permet de débattre de nouvelles options discutées par la communauté. Il est toujours guidé par Kuwako Toshio - ce dernier étant extérieur au groupe et non impliqué dans le cadre du projet à titre individuel, statut garantissant sa neutralité -, ce qui permet de donner une fluidité au débat sans qu'aucun blocage ne vienne arrêter les négociations. Grâce à cette spirale d'échange, chaque proposition n'est jamais imposée mais acceptée en connaissance de cause et donc assumée par la communauté locale. On peut ainsi parler de territorialisation durable.

\section{Une médiation profitable}

Le nouveau plan proposé pour la rue commerçante du sanctuaire d'Izumo consiste à réduire la chaussée. Ralentissant la vitesse des automobiles, il libère un véritable espace piétonnier permettant aux habitants et aux touristes de s'emparer de l'espace public (figure 8). Les choix concernant le pavage, l'éclairage et les divers matériaux propres à l'aménagement urbain de tels espaces seront proposés et choisis en toute impartialité par l'ensemble des habitants. Jusqu'à la fin du processus, ceux-ci peuvent émettre leurs doutes ou faire part de leur ignorance concernant certaines données, ce qui peut justifier telle ou telle nouvelle orientation que les experts seront dans l'obligation de clarifier, voire de remplacer par de nouvelles propositions.

Figure 6. Proposition initiale ayant un impact sur le bâti

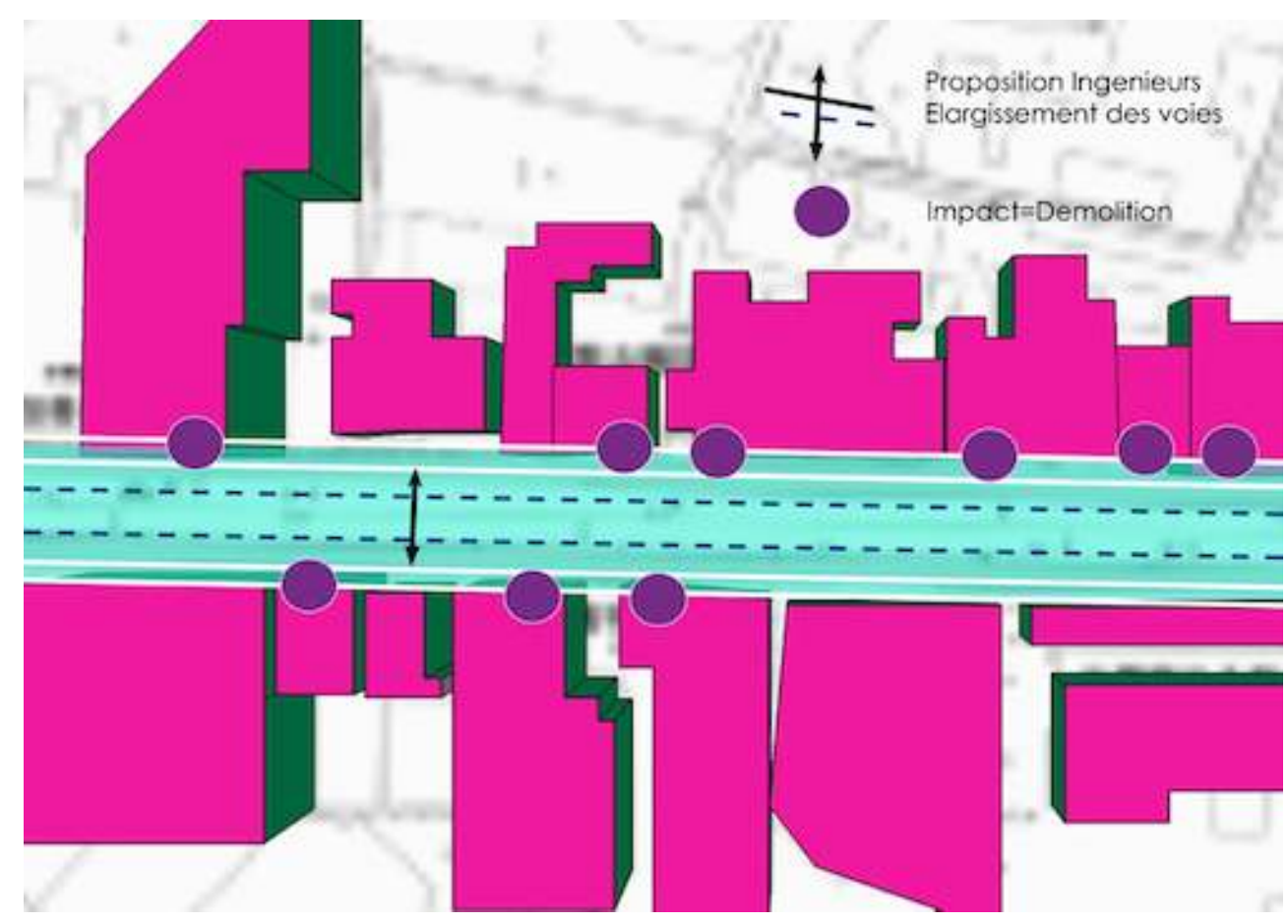

Source : Alban Mannisi. 
Figure 7. Proposition de redistribution des flux de la chaussée présentée par l'équipe de paysagiste après consultation des habitants

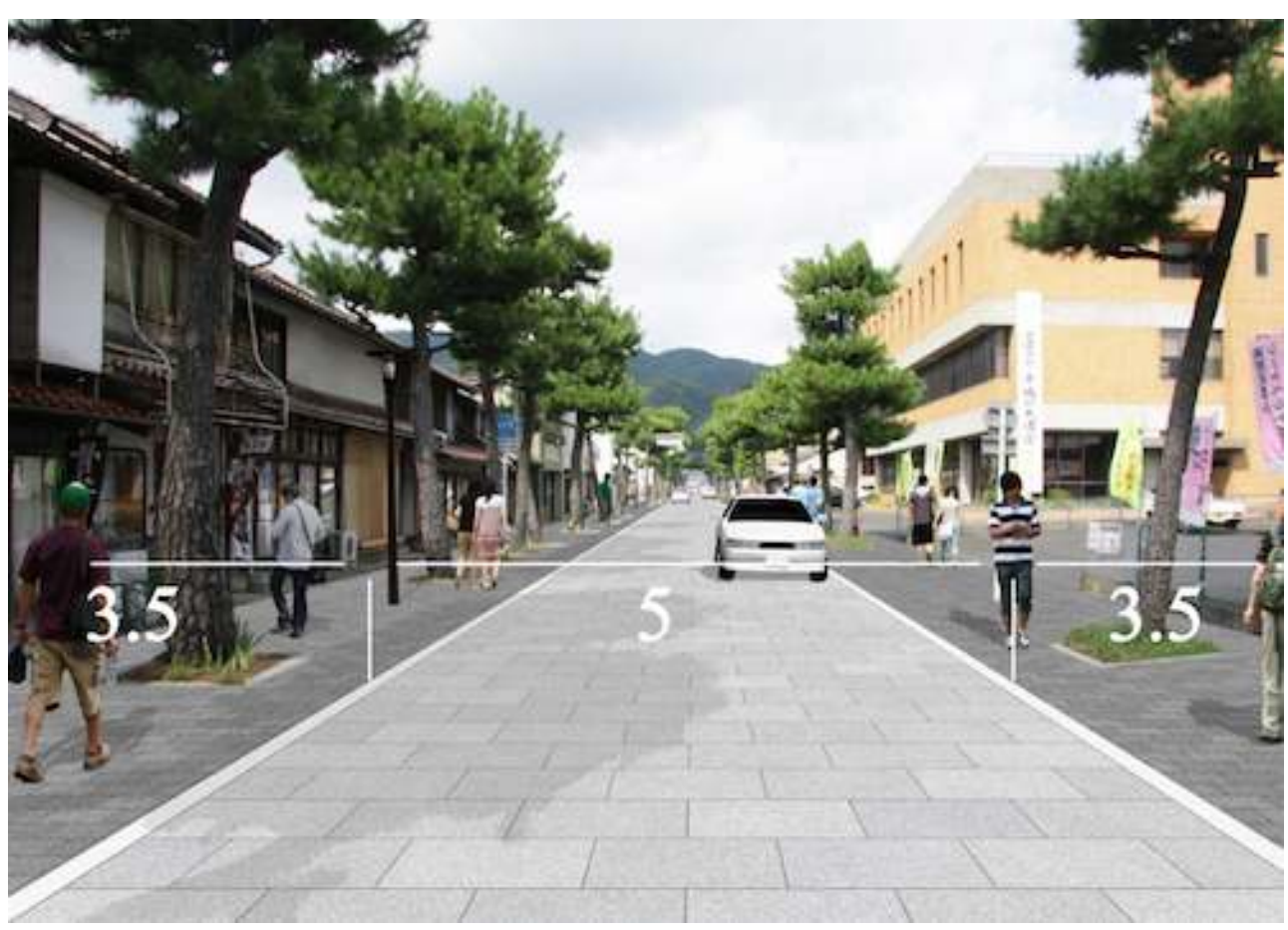

Source : Préfecture de Shimane.

Figure 8. Dès le nouveau partage au sol des chaussées piétonnes et automobiles, les habitants s'emparent de la rue commerçante

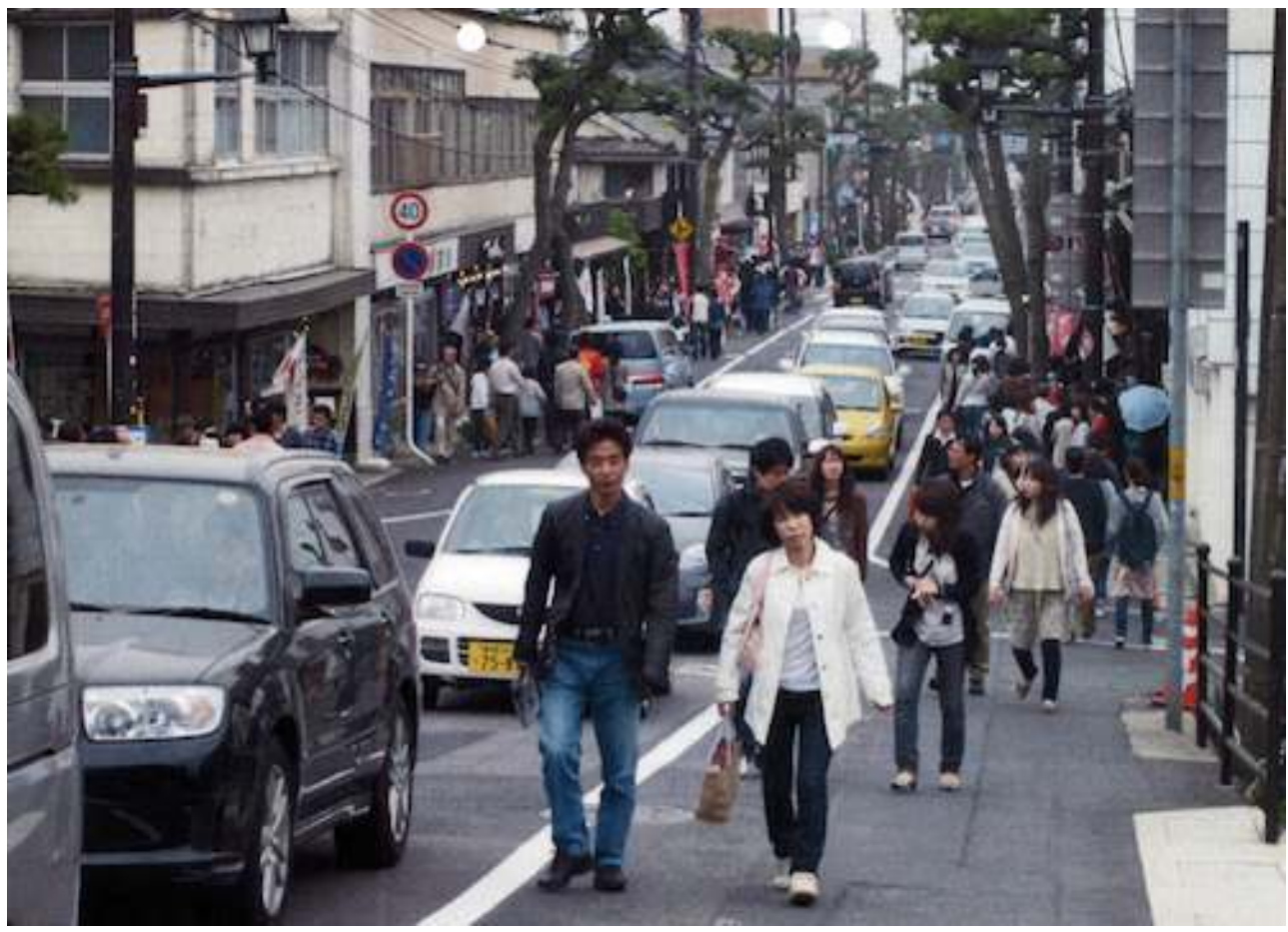

Source : Préfecture de Shimane. 


\section{Conclusion}

L'importance donnée à de tels projets participatifs est révélatrice de bien des difficultés que porte en lui l'usage d'un territoire. L'intérêt de la démarche de Kuwako Toshio, simple mais difficile à mener de bout en bout, réside dans le fait qu'elle s'appuie sur un large mouvement de resolidarisation des sociétés au cœur des problématiques environnementales, en reformulant de nouvelles procédures du vivre ensemble. La lecture dynamique du lieu du kûkan no rireki permet non de nier le principe multiscalaire opérant dans la construction d'un milieu de vie, mais de l'éclairer pour puiser dans ses forces. Ainsi la démarche de médiation du philosophe enrichit l'émergence de nouvelles pratiques de construction de paysages partagés. Il ne s'agit plus de parfaire l'image qu'une société a d'elle-même dans l'esthétisation de son modelage territorial (Nadai, 2007), mais bien de refonder un environnement social plus ou aussi riche qu'avant.

27 Avec le kûkan no rireki, Kuwako Toshio revisite le lien entre les Japonais et leur rapport avec la nature (Berque, 1986). Le rôle de médiateur qu'il occupe, semblable à ce qui existe désormais en France $^{15}$, rétablit le lien détruit entre une société frappée d'incompréhension et les aménageurs de son environnement humain et non humain ${ }^{16}$.

Actuellement, l'idée de paysage suscite un engouement international. Le thème catalyse en effet les maux issus de la territorialisation moderne, mais donne essentiellement la parole aux aménageurs paysagistes ou aux rares médiateurs chargés de résoudre les conflits environnementaux. Et l'usager dans tout cela? Et le citoyen acteur de son environnement?

C'est ce que tente de démontrer la théorie de l'acteur réseau ${ }^{17}$ développée par Bruno Latour et Michel Callon (Callon, Latour, 1981). Thèse qui dépasse l'instrumentalisation équivoque de la société civile afin de contourner de coûteuses controverses et de remettre au centre des débats la soutenabilité des espaces de vie. Car l'individu agit au sein de sa communauté : «il ne s'agit pas de "faire voter" le citoyen, mais d'inventer des dispositifs tels que ceux de ces citoyens dont parlent les experts scientifiques puissent être effectivement présents, capables de poser les questions auxquelles leur intérêt les rend sensibles, d'exiger des explications, de poser des conditions, de suggérer des modalités, bref de participer à l'invention »(Stengers, 1993).

Nos remerciements à Kuwako Toshio à et son équipe du Laboratoire d'Ethique Environnementale de l'Université Technologique de Tokyo.

\section{BIBLIOGRAPHIE}

Augé, M., Non-Lieux. Introduction à une anthropologie de la surmodernité, Paris, Éditions du Seuil, 1992, p. 99.

Berger, P., Luckmann, T., La Construction sociale de la réalité (1966), Paris, Armand Colin, 2006. 
Berque, A., Le Sauvage et Artifice. Les Japonais devant la nature, Paris, Gallimard, 1986.

Callon, M., Lascousmes, P., Barthes, Y., Agir dans un monde incertain, essai sur la démocratie technique, Paris, Éditions du Seuil, 2001, p. 163.

Callon, M.,et Latour, B. « Unscrewing the Big Leviathan : How Actors Macrostructure Reality and How Sociologists Help Them To Do So ", dans Knorr, K. et Cicourel, A. (dir.), Advances in Social Theory and Methodology : Toward an Integration of Micro- and Macro-Sociologies, Boston, Routledge and Kegan Paul, 1981, p. 277-303.

Dewey, J., Le Public et ses problèmes (1927), Paris, Gallimard, coll. « Folio essais », 2005.

Frémont, A., La Région, espace vécu, Paris, Flammarion, 1976.

Gauchet, M., Le Goff, J.-P., Bourg, D. et Whiteside, K., « Autour de : vers une démocratie écologique ", Le Débat, nº 164, février 2011, p. 144-172.

Heidegger, M., Etre et Temps (1927), Paris, Gallimard, 1986, chap. V, § 75.

Kuwako, T., « Ancient Landscape and Project Management of Participatory Processes Concerning Public Works », ERA Arqueologia, 6e conférence annuelle, Portugal, 2 mars 2007, p. 15-18.

Kuwako, T., Kisō no Tetsugaku (« Philosophie du changement et de la transformation »), Tōkyō, Shinyosha, 1996.

Kuwako, T., Kankyô no Tetsugaku (« Une philosophie de l'environnement »), Tōkyō, Kodansha Gakujutsu Bunko, 1999a.

Kuwako, T., Saigyô no Fûkei (Les Paysages de Saigyô), Tōkyō, NHK Books, $1999 b$.

Kuwako. T., « Kanmin Gouikeiseiron (“Théorie pour un gouvernement citoyen") », The Journal of Survey, $\mathrm{n}^{\circ} 7,2008$, p. 27-30.

Kuwako, T., « Komyunikeeshon ni okeru Kansei to Gouikeisei (Construction du consensus et sensibilité au territoire en communication) », The Journal of the Institute of Electronics, Information and Communication Engineers, Denshi Jouhou Tsuushin Gakkai, novembre 2009, p. 967-969.

Latour, B., Nous n'avons jamais été modernes. Essai d'anthropologie symétrique, Paris, La Découverte, 1991, p. 194.

Mannisi, A., «La résurgence des sociétés civiles urbaines au Japon. Éléments pour une résilience territoriale. », Croisements, $\mathrm{n}^{\circ}$ 2, Paris, Atelier des Cahiers, 2012, p. 178-198.

Mermet, L. (sous la dir. de), Étudier des écologies futures. Un chantier ouvert pour les recherches prospectives environnementales, Bruxelles, Bern, Berlin, Frankfurt am Main, New York, Oxford, Wien, Peter Lang, coll. « EcoPolis », 2005.

Nadai, A., « Degré zéro. Portée et limites de la théorie de l'artialisation dans la perspective d'une politique du paysage ", Cahiers de géographie du Québec, $\mathrm{n}^{\circ}$ 144, 2007, p. 333-343.

Ollagnon, H., « La gestion de la biodiversité : quelles stratégies patrimoniales ? », Responsabilité et Environnement, $\mathrm{n}^{\circ} 44$, octobre 2006, p. 50-56.

Rancière, J., Aux bords du politique, Paris, Gallimard, 1998, p. 36.

Roger, A. (sous la dir. de), La Théorie du paysage en France, Paris, Champs Vallon, 1995, p. 42-73.

Stengers, I., L'Invention des sciences modernes, Paris, La Découverte, 1993, p. 180.

Susskind, L., Dealing with an Angry Public. The Mutual Gains Approach to Resolving Disputes, New York, Free Press, 2010. 
Tricot, A., « La prise en compte de la controverse dans l'aménagement du territoire : peut-on négocier le futur? », Espaces et Sociétés, n 74-75, 1994, p. 69-97.

\section{NOTES}

1. Principe 10 de la déclaration de Rio ; voir le site www.unep.org.

2. Parmi celles-ci, citons la loi Bouchardeau (1983) sur la démocratisation des enquêtes publiques, la convention d'Aarhus (1998) sur la participation du public au processus décisionnel, la loi Barnier (1995) instituant la consultation du public et des associations en amont des décisions d'aménagement, la loi Voynet (1999) dite la loi d'orientation sur l'aménagement et le développement durable du territoire - cette dernière favorisant une meilleure concertation autour des projets d'aménagement, et d'élargissement des possibilités de débat autour des projets publics -, la loi SRU (2000) qui impose la nécessité d'une concertation avant l'élaboration ou la révision d'un schéma de cohérence territoriale ou d'un plan local d'urbanisme dans une perspective de développement durable, ou encore la loi Vaillant (2002) sur la démocratie de proximité favorisant l'émergence de conseils de quartier pour les communes de plus quatre-vingt mille habitants.

3. Voir le très intéressant dossier de la revue Le Débat et les controverses que ce projet suscite (Gauchet, M., Le Goff, J.P., Bourg, D. et Whiteside, K. 2011).

4. NPO pour Non Profitable Organisation. Le terme anglo-saxon est employé au Japon et correspond aux associations à but non lucratif françaises.

5. L'article 6 précise ainsi la nécessaire participation active des citoyens et leur implication au cœur des projets gouvernementaux développés à l'échelle locale. La décentralisation étant un des points forts de la politique libérale japonaise, l'aménagement du territoire profite ainsi de cette responsabilisation de la société civile.

6. Un autre cas d'étude du philosophe est développé dans l'article de la revue Croisements (Mannisi, 2012).

7. Dans le cadre de la médiation aux États-Unis, l'urbaniste Laurence Susskind, au sein de son laboratoire de recherche de planification urbaine du Massachusetts Institute of Technology, fait figure de modèle au Japon.

8. En relation avec le livre fondateur du mouvement de sociologie constructiviste de Peter Berger et Thomas Luckmann (1966).

La nature est considérée au Japon comme «sacrée en elle-même », en dehors de toute croyance théiste ou scientiste. D'où un discours «constructiviste" socialement plus orienté vers les relations de l'homme à son environnement.

9. Pour une différenciation entre le et la politique (Rancière, 1998).

10. Formule désormais employée, comme "forums hybrides", pour évoquer les projets participatifs (Callon, M. Lascousmes, P., Barthes, Y., 2001).

11. La ville d'Izumo, qui s'étend autour d'un bras de rivière, connaît de très fortes inondations. Les différents projets pour les résorber ont échoué. Les habitants ont désormais vis-à-vis des nouveaux projets d'experts une forte défiance et posent des vetos qui ralentissent considérablement les nouveaux plans d'aménagement. C'est donc pour éviter ces ralentissements que la ville a fait appel à un médiateur.

12. Le spectacle touristique des monuments, comme le spectacle des inondations, est médiatisé en tant que « paysage spectacle » qui explique l'intérêt du gouvernement pour certains paysages. Sur la notion de paysage spectacle, lire de Yves Lacoste, «À quoi sert le paysage ? Qu'est ce que le beau paysage?» (Roger, 1995). 
13. La consultation des citoyens est une procédure obligatoire dans les projets d'aménagement au Japon depuis 1968 (nouvelle loi d'urbanisme). D'ordinaire sans hiatus, ici les habitants vont poser un véto systématique à chaque étape de validation des propositions.

14. Composé de représentants des habitants de la rue, des ingénieurs en charge du projet incriminé, de nouvelles équipes d'urbanistes paysagistes mandatés par la ville et du médiateur Kuwako Toshio.

15. Il existe aujourd'hui une formation en master "Paysages et médiations " à l'université d'Angers/École du paysage d'Angers (INHP-Agrocampus Ouest).

16. Voir le chapitre « Le parlement des choses » (Latour, 1991).

17. Dite Actor Network Theory (ANT) car élaborée depuis une vision globale.

\section{RÉSUMÉS}

Suite au désarroi des sociétés civiles dû à leur perte de confiance envers le politique et les experts en aménagement du territoire, les acteurs de la construction du paysage ont depuis mis en œuvre des procédures complexes afin d'établir des protocoles de projets au plus près des besoins des habitants des espaces investis. Dans un Japon contemporain où la brutalité de l'aménagement du territoire a bouleversé le rapport à la nature, nous rendons compte de l'expérience du philosophe Kuwako Toshio aux prises avec des problèmes d'aménagement du territoire. Ce dernier développe des méthodes de médiation environnementale afin de mieux associer les habitants à leur milieu.

Following the confusion of civil societies due to their loss of confidence in politics and planning experts, those involved in landscape construction have since implemented procedures to establish complex protocols of projects closer to the needs of residents of invested spaces. In a contemporary Japan, where the brutality of planning has changed the relationship with nature, we will report the experience of the philosopher Kuwako Toshio. Engage with territorial Planning issues, he develops methods of environmental mediation to better connect people to their environment.

\section{INDEX}

Keywords : citizen participation, Eenvironmental mediation, Japan, social construction of the reality, spatial portrait

Mots-clés : médiation environnementale, construction sociale de la réalité, Japon, participation citoyenne, portrait du lieu

\section{AUTEUR}

\section{ALBAN MANNISI}

Architecte paysagiste, il vit au Japon où il a installé son agence SEIWOOO. Il est chercheur associé des laboratoires d'éthique environnementale de l'université technologique de Tōkyō, de l'unité de planification urbaine et territoriale de l'université de Tōkyō et doctorant au laboratoire 
Architecture/Milieu/Paysage de l'École d'architecture de Paris La Villette.

mannisialban[at]gmail[dot]com 